\title{
On the Utility of the Inverse Gamma Distribution in Modeling Composite Fading Channels
}

\author{
Pablo Ramírez-Espinosa and F. Javier Lopez-Martinez \\ Dpto. de Ingeniería de Comunicaciones, ETSI Telecomunicacion \\ Universidad de Málaga, Málaga, E-29071, Spain \\ email: \{pre, fjlopezm\}@ic.uma.es
}

\begin{abstract}
We introduce a general approach to characterize composite fading models based on inverse gamma (IG) shadowing. We first determine to what extent the IG distribution is an adequate choice for modeling shadow fading, by means of a comprehensive test with field measurements and other distributions conventionally used for this purpose. Then, we prove that the probability density function and cumulative density function of any IG-based composite fading model are directly expressed in terms of a Laplace-domain statistic of the underlying fast fading model, and in some relevant cases, as a mixture of well-known state-of-the-art distributions. We exemplify our approach by presenting a composite IG/two-wave with diffuse power fading model, for which its statistical characterization is directly obtained in a simple form.
\end{abstract}

Index Terms-Shadowing, fading, inverse gamma distribution, composite fading models.

\section{INTRODUCTION}

In wireless channels, the random fluctuations affecting the radio signals have been classically divided into two types: fast fading, as a result of the multipath propagation, and shadow fading or shadowing, which is caused by the presence of large objects like trees or buildings. Aiming to analyze and improve the performance of wireless communication systems, considerable efforts have been devoted to the characterization of these two effects. For instance, several models are used to describe the statistical behavior of fast fading, including both the classical ones such as Rayleigh, Rice, and Nakagami-m [1-3] as well as generalized models [4, 5]. With respect to shadow fading, the lognormal distribution is widely accepted as the right choice $[2,6]$, supported by empirical verification.

In practice, both fast fading and shadowing occur simultaneously, although at different time scales. Therefore, composite fading models arise to characterize the combined impact of these two effects. In the literature, there are two different alternatives to incorporate the effect of shadowing on the top of fading: line-of-sight (LoS) shadowing, where the shadowing only affects the specular component [7], and multiplicative shadowing, in which both the specular and scattered fading components are shadowed [8]. Examples of the first type of composite models are the Rician shadowed [7], the $\kappa-\mu$ shadowed [9] and the fluctuating Beckmann [10]. On the other hand, multiplicative shadowing composite models originally

This work has been funded by the Spanish Government and the European Fund for Regional Development FEDER (project TEC2017-87913-R) and by Universidad de Malaga. arose as a combination of lognormally-distributed shadowing and classical fading models like Rayleigh or Nakagami- $m$ $[2,11]$. However, all these models inherit the complicated formulation of the lognormal distribution, considerably limiting their usefulness for further analytical calculations.

Alternatively to the lognormal distribution, the gamma distribution has also been proposed in the literature, showing its suitability to model shadowing through goodness-of-fit tests $[12,13]$. Due to its mathematical tractability, new composite models arose as a combination of gamma shadowing and different fast fading distributions, e.g. the $K$ distribution (Gamma/Rayleigh) [14], Gamma/Weibull [15], Gamma/ $\kappa-\mu$ and Gamma/ $\eta-\mu$ [16]. A double shadowing model combining LoS fluctuation due to human-body shadowing with multiplicative shadowing was recently introduced [17]. A different alternative to model shadowing is given in [18-20], where the inverse Gaussian distribution is proposed; this approach is specially accurate to approximate the lognormal distribution when the variance of shadowing is very large. In the recent years, the inverse gamma (IG) distribution has been used to characterize shadowing based upon the fact that it admits a relatively simple mathematical formulation. Based on the IG distribution, different composite models have been proposed $[8,21]$. However, a rigorous empirical validation to assess the adequacy of the IG distribution to model shadow fading has not been performed in depth. To the best our knowledge, this has only been partially addressed in [22].

In this work, we aim to find answer to two key questions: (a) is the use of the IG distribution to model shadowing supported by practical evidences?, and (b) does the IG distribution bring additional benefits to other alternatives? In order to answer the first one, we perform an extensive set of goodness-of-fit tests using empirical data measurements. Once this is accomplished, we present a general approach to the statistical characterization of composite fading channels with IG shadowing. Notably, we show that the probability density function (PDF) and the cumulative distribution function (CDF) of the composite fading distribution can be directly expressed in terms of a generalization of the moment generating function (MGF) of the fast fading model. This holds for any arbitrary choice of fading distribution, and allows to use existing results in the literature to fully characterize the statistics of the composite fading model. 


\section{EMPIRICAL VALIDATION OF IG SHADOWING}

\section{A. Definitions of shadowing distributions}

Definition 1 (Lognormal distribution): Let $X$ be a random variable (RV) following a Gaussian distribution with mean $\mu$ and variance $\sigma^{2}$. Then, the $\operatorname{RV} Y=e^{X}$ is lognormally distributed with $\mathrm{CDF}$

$$
F_{Y}^{\mathrm{L}}(\mu, \sigma ; y)=\frac{1}{2}+\frac{1}{2} \operatorname{erf}\left(\frac{\ln y-\mu}{\sqrt{2 \sigma^{2}}}\right)
$$

where $\operatorname{erf}(\cdot)$ is the error function [23, eq. (7.1.1)].

Definition 2 (Gamma distribution): Let $Y$ be a RV following a gamma distribution with shape parameter $k$ and $\mathbb{E}[Y]=\Omega$. Then, the PDF and CDF of $Y$ are given by

$$
\begin{aligned}
& f_{Y}^{\mathrm{G}}(k, \Omega ; y)=\frac{k^{k}}{\Gamma(k) \Omega^{k}} y^{k-1} e^{-k y / \Omega}, \\
& F_{Y}^{\mathrm{G}}(k, \Omega ; y)=\frac{1}{\Gamma(k)} \gamma(k, k y / \Omega),
\end{aligned}
$$

with $\Gamma(\cdot)$ and $\gamma(\cdot, \cdot)$ the gamma function and the lower incomplete gamma function, respectively [23, eqs. (6.1.1) and (6.5.2)].

Definition 3 (Inverse Gaussian distribution): Let $Y$ be a RV following an inverse Gaussian distribution with parameters $\mu_{I}$ and $\lambda$. Then, the CDF of $Y$ is given by

$$
\begin{aligned}
F_{Y}^{\mathrm{IG}}\left(\mu_{I}, \lambda ; y\right)= & \frac{1}{2}+\frac{1}{2} \operatorname{erf}\left(\sqrt{\frac{\lambda}{2 y}}\left(\frac{y}{\mu_{I}}-1\right)\right)+\exp \left(\frac{2 \lambda}{\mu_{I}}\right) \\
& \times\left[\frac{1}{2}+\frac{1}{2} \operatorname{erf}\left(-\sqrt{\frac{\lambda}{2 y}}\left(\frac{y}{\mu_{I}}+1\right)\right)\right] . \text { (4) }
\end{aligned}
$$

Definition 4 (Inverse gamma distribution): Let $Y$ be a RV following an IG distribution with shape parameter $\alpha$ and $\mathbb{E}[Y]=\Omega_{i}$. Then, the PDF and CDF of $Y$ are given by

$$
\begin{aligned}
& f_{Y}^{\mathrm{Ig}}\left(\alpha, \Omega_{i} ; y\right)=\frac{\Omega_{i}^{\alpha}(\alpha-1)^{\alpha}}{\Gamma(\alpha)} y^{-\alpha-1} e^{-\Omega_{i}(\alpha-1) / y}, \\
& F_{Y}^{\mathrm{Ig}}\left(\alpha, \Omega_{i} ; y\right)=\frac{1}{\Gamma(k)} \Gamma\left(k, \Omega_{i}(\alpha-1) / y\right),
\end{aligned}
$$

where $\Gamma(\cdot, \cdot)$ is the upper incomplete gamma function [23, eq. (6.5.3)].

\section{B. Fitting to field measurements}

In order to check the suitability of the inverse gamma distribution to model shadowing, we here compare empirical CDFs obtained from data measurements in different scenarios with the different models defined in the previous subsection, i.e. lognormal, gamma, inverse Gaussian and inverse gamma. Specifically, the Cramer-von Mises test is used for the comparison, which is a more powerful option than the well-known Kolmogorov-Smirnov test, i.e. the probability of accepting the alternative hypothesis when the alternative hypothesis is true is higher in the Cramer-von Mises test [13, 24]. It is defined as the integrated mean square error between the empirical CDF, $\widehat{F}_{\xi}(x)$, and the theoretical one, $F_{\xi}(x)$, i.e.

$$
\omega^{2}=\int_{-\infty}^{\infty}\left|\widehat{F}_{\xi}(x)-F_{\xi}(x)\right|^{2} d x
$$

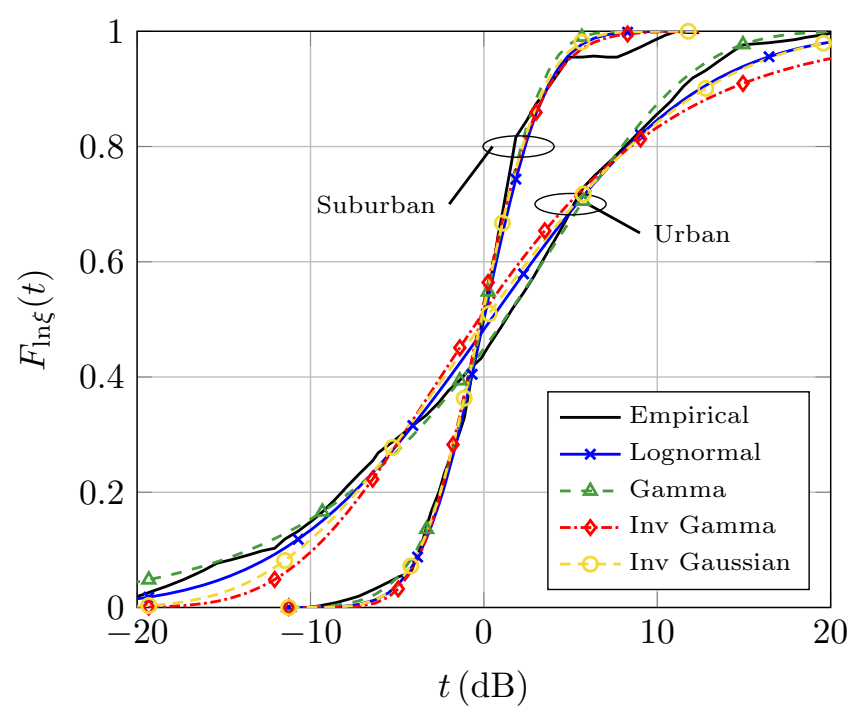

Fig. 1. Shadowing CDF for data in [25, Fig. 4], corresponding to urban and suburban scenarios at $169 \mathrm{MHz}$. Parameters for urban scenario: lognormal ( $\mu=0.0476, \sigma=1.083)$, gamma $(k=1.15, \Omega=1.572)$, inverse gamma $\left(\alpha=1.18, \Omega_{i}=4.65\right)$ and inverse Gaussian $\left(\mu_{i}=1.864, \lambda=1.041\right)$. Parameters for suburban scenario: lognormal $(\mu=0, \sigma=0.325)$, gamma ( $k=10.14, \Omega=1.014)$, inverse gamma $\left(\alpha=9.8, \Omega_{i}=1.05\right)$ and inverse Gaussian $\left(\mu_{i}=1.036, \lambda=9.573\right)$.

TABLE I

Results of the CRAméR-VON Mises test, $\omega^{2}$, FOR DATA IN Figs. 1-3. SHOWED VALUES ARE NORMALIZED BY $10^{-3}$.

\begin{tabular}{|c||c|c|c|c|}
\hline$\omega^{2} \times 10^{3}$ & Lognormal & Gamma & Inv. Gamma & Inv. Gaussian \\
\hline \hline Fig. 1 urban & 2.255 & 1.057 & 10.59 & 4.908 \\
\hline Fig. 1 suburban & 1.291 & 1.182 & 1.072 & 1.094 \\
\hline Fig. 2 $(a)$ & 2.941 & 5.499 & 1.521 & 2.607 \\
\hline Fig. 2 $(b)$ & 1.627 & 1.100 & 2.400 & 1.713 \\
\hline Fig. 3 & 0.0429 & 0.0224 & 0.0215 & 0.0220 \\
\hline
\end{tabular}

Aiming to cover a wide variety of propagation environments, empirical distributions have been obtained from data measurements in three different scenarios: urban and suburban scenarios for smart wireless metering systems at $169 \mathrm{MHz}$ [25], suburban at $910.25 \mathrm{MHz}$ [14] and indoor scenarios at 26 $\mathrm{GHz}$ (mmWave) [26]. Since shadowing data are usually given in logarithmic scale (typically as deviations over the path loss in $\mathrm{dB}$ ), the fitting is not performed over the shadowing random variable (RV) $\xi$ but over $\ln \xi$. Therefore, the corresponding change of variables is required in the theoretical CDFs in Definitions 1-4. Taking that into account, the empirical and theoretical CDFs for each scenario are depicted in Figs. 1-3, and the results for the Cramer-von Mises test are shown in Table I, while the distribution parameters for each case are detailed in the figure captions.

From Table I, the use of inverse gamma distribution to model shadowing seems a reasonable choice, or at least as much as the gamma or lognormal distributions. In fact, it provides the most accurate fitting to the empirical CDF in three 


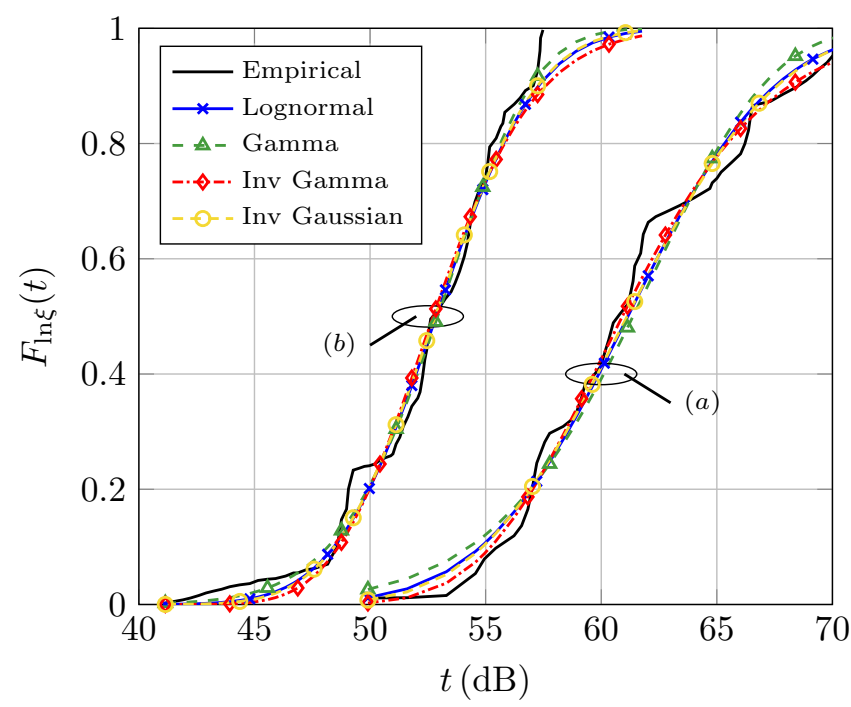

Fig. 2. Shadowing CDF data in [14, Fig. 1-2], corresponding to suburban scenarios at $910.25 \mathrm{MHz}$. Parameters for case $(a)$ : lognormal $(\mu=7.05$, $\sigma=0.572)$, gamma $(k=3.448, \Omega=1300)$, inverse gamma $(\alpha=3.315$, $\left.\Omega_{i}=1440\right)$ and inverse Gaussian $\left(\mu_{i}=1351.9, \lambda=3620.3\right)$. Parameters for case $(b)$ : lognormal $(\mu=6.09, \sigma=0.398)$, gamma $(k=6.792, \Omega=$ 467.36), inverse gamma $\left(\alpha=6.54, \Omega_{i}=487.36\right)$ and inverse Gaussian $\left(\mu_{i}=475.38, \lambda=2856.3\right)$.

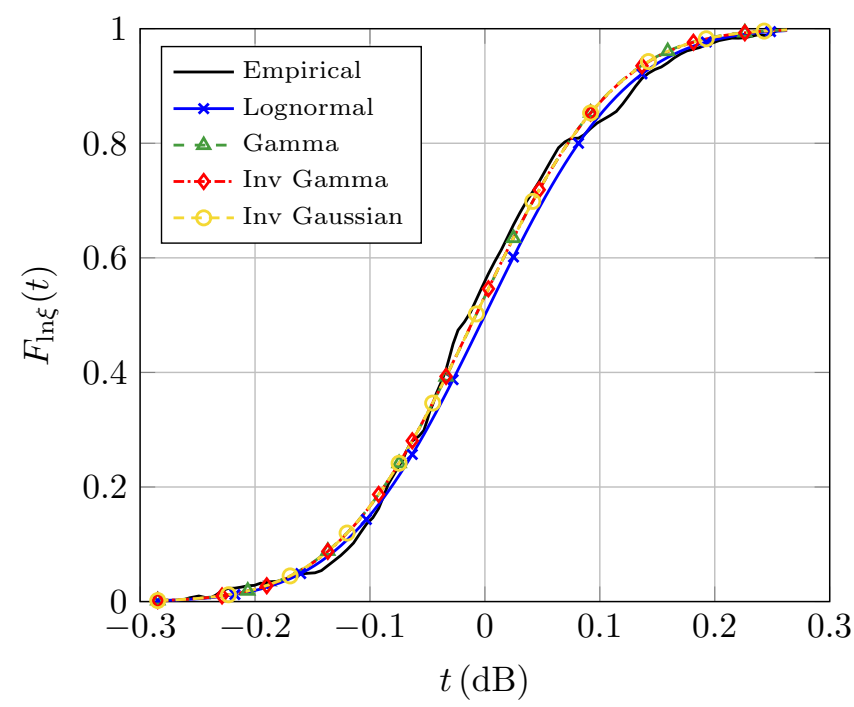

Fig. 3. Shadowing CDF for data in [26, Fig. 3b], corresponding to an indoor scenario at $26 \mathrm{GHz}$. Parameters: lognormal $(\mu=0, \sigma=0.011)$, gamma ( $k=8319, \Omega=0.998)$, inverse gamma $\left(\alpha=8316, \Omega_{i}=1\right)$ and inverse Gaussian $\left(\mu_{i}=1, \lambda=8310\right)$.

of the cases under analysis, whilst the widely used lognormal distribution is always outperformed by either the gamma or the inverse gamma models. Note also that in the indoor mmWave scenario (Fig. 3), there are little differences between these models due to the low variance of shadowing measurements.

Based on these results, inverse gamma composite models are justified, since there does not seem to be any major drawback in using the inverse gamma distribution instead of the other alternatives. Most importantly, its mathematical tractability will lead to simpler expressions for the main statistics of composite models than those resulting from considering lognormal, gamma or inverse Gaussian shadowing. This will be now formally stated in the following section.

\section{STATISTICAL CHARACTERIZATION OF INVERSE GAMMA COMPOSITE FADING MODELS}

\section{A. Physical model}

Consider a RV $W$ characterizing the instantaneous received signal power in a multipath propagation scenario affected by both shadowing and fast fading. Then, $W$ can be expressed as

$$
W=\Omega \widehat{\xi} \widehat{X}
$$

where $\Omega$ is the mean signal power and $\widehat{\xi}$ and $\widehat{X}$ are independent RVs representing respectively the shadowing and the fast fading, with $\mathbb{E}[\widehat{\xi}]=\Omega_{\xi}$ and $\mathbb{E}[\widehat{X}]=\Omega_{X}$. Due to its mathematical tractability and its suitability to model shadowing, we consider that $\widehat{\xi}$ is inverse gamma distributed with shape parameter $m$ and $\operatorname{PDF} f_{\widehat{\xi}}^{\mathrm{Ig}}\left(m, \Omega_{\xi} ; t\right)$ given in (5), while $\widehat{X}$ follows any arbitrary fading distribution. For the sake of simplicity, $W$ can be rewritten as

$$
W=\bar{W} \xi X
$$

where $\bar{W} \triangleq \mathbb{E}[W]=\Omega \Omega_{\xi} \Omega_{X}$ and $\xi$ and $X$ are the normalized versions of $\widehat{\xi}$ and $\widehat{X}$, i.e. $\xi$ is an inverse gamma $\mathrm{RV}$ with shape parameter $m$ and $\mathbb{E}[\xi]=1$ and $X$ follows any fading distribution with $\mathbb{E}[X]=1$. Note that the value of $m$ is directly related to the severity of shadowing. Smaller values of $m$ mean that the variance of the IG distribution is larger, while large values of $m$ render less spread values of $\xi$. This can also be observed from the parameters used in Figs. 1-3.

\section{B. Statistical characterization}

We aim to characterize the composite fading model in (9), giving general expressions for its main statistics in terms of those of the underlying fading model $X$. Specifically, we will show that the PDF and CDF of $W$ can be readily obtained from the generalized moment generating function (GMGF) of $X$, which is defined below.

Definition 5 (GMGF): Let $X$ be a continuous nonnegative RV with PDF $f_{X}(x)$. Then the GMGF of $X$ is defined as

$$
\phi_{X}^{(n)}(s) \triangleq \mathbb{E}\left[X^{n} e^{X s}\right]=\int_{0}^{\infty} x^{n} e^{x s} f_{X}(x) d x .
$$

Note that, if $n \in \mathbb{N}^{+}$, then the GMGF coincides with the $n^{t h}$ order derivative of the moment generating function (MGF), defined as $M_{X}(s)=\mathbb{E}\left[e^{s X}\right]=\phi_{X}^{(0)}(s)$.

With Definition 5, we now calculate the PDF and CDF of $W$ in the following lemmas.

Lemma 1: Let $W$ be a RV characterizing the instantaneous received signal power as in (9). Then, for $m>1$, its PDF is given by

$$
f_{W}(u)=\frac{\bar{W}^{m}(m-1)^{m}}{u^{m+1} \Gamma(m)} \phi_{X}^{(m)}\left(\frac{(1-m) \bar{W}}{u}\right)
$$


where $\phi_{X}^{(m)}(\cdot)$ is the GMGF of $X$ in (10).

Proof: When conditioned on $\xi$, the PDF of $W$ is

$$
f_{W}(u \mid \xi)=\frac{1}{\bar{W} \xi} f_{X}\left(\frac{u}{\bar{W} \xi}\right),
$$

with $f_{X}(\cdot)$ the PDF of $X$. The unconditional PDF of $W$ is therefore obtained by averaging on $1 / \xi$ as

$$
f_{W}(u)=\int_{0}^{\infty} \frac{1}{\bar{W}} t f_{X}\left(\frac{u t}{\bar{W}}\right) f_{1 / \xi}(t) d t
$$

where, since $\xi$ is inverse gamma distributed then $1 / \xi$ is gamma distributed with PDF $f_{1 / \xi}^{\mathrm{G}}(m, m /(m-1) ; t)$. Therefore, substituting in (13) and performing the change of variables $y=u t / \bar{W}$ lead to

$$
f_{W}(u)=\frac{[\bar{W}(m-1)]^{m}}{u^{m+1} \Gamma(m)} \int_{0}^{\infty} f_{X}(y) y^{m} e^{(1-m) \bar{W} y / u} d y .
$$

The above integral correspond to the GMGF of $X$ evaluated at $s=(1-m) \bar{W} / u$, obtaining (11) and completing the proof.

Lemma 2: Let $W$ be a RV characterizing the instantaneous received signal power as in (9). Then, for $m>1$, its CDF is given by

$$
F_{W}(u)=1-\sum_{n=0}^{\infty} \frac{[\bar{W}(m-1)]^{m+n}}{u^{m+n} \Gamma(m+n+1)} \phi_{X}^{(m+n)}\left(\frac{(1-m)}{u \bar{W}^{-1}}\right) .
$$

Proof: Similarly to the PDF, the CDF of $W$ can be calculated as

$$
F_{W}(u)=\int_{0}^{\infty} F_{X}\left(\frac{u t}{\bar{W}}\right) f_{1 / \xi}(t) d t .
$$

Performing the change of variables $y=u t / \bar{W}$ and integrating by parts we obtain

$$
F_{W}(u)=1-\int_{0}^{\infty} F_{1 / \xi}\left(\frac{\bar{W}}{u} y\right) f_{X}(y) d y,
$$

where $F_{1 / \xi}(\cdot)$ is the CDF of $1 / \xi$, which is gamma distributed with shape parameter $m$ and $\Omega=m /(m-1)$. Therefore, using (3) and [23, eqs. (6.5.4) and (6.5.24)] we can rewrite (17) as

$$
\begin{aligned}
F_{W}(u)= & -\sum_{n=0}^{\infty} \frac{[\bar{W}(m-1)]^{m+n}}{u^{m+n} \Gamma(m+n+1)} \\
& \times \int_{0}^{\infty} y^{m+n} e^{(1-m) \bar{W} y / u} f_{X}(y) d y,
\end{aligned}
$$

where the integral corresponds to the GMGF of $X$ given in (10), yielding (15).

Lemmas 1 and 2 provide general expressions for the PDF and CDF of $W$ in terms of the GMGF of the underlying fading model, unifying the statistical analysis of inverse gamma composite fading models. Indeed, the analytical tractability of these statistics will strongly depend on the ability to calculate the GMGF of $X$. In the most general case where the GMGF of $X$ is unknown or has an intractable form, the integral in (10) can be computed numerically, as it is generally well-behaved since the exponential term should ensure the convergence.

For many fading models, the GMGF can be obtained in closed form for arbitrary $m$. This is the case of the very general $\kappa-\mu$ shadowed distribution [9] (which includes most popular fading distributions as special cases), for which the GMGF can be readily obtained from [27, eq. (11)] as

$$
\begin{aligned}
\phi_{\kappa-\mu \mathcal{S}}^{(p)}(s)= & \frac{(\mu)_{p} \mu^{\mu} \underline{\underline{m}} \underline{m}^{\underline{m}}(1+\kappa)^{\mu}}{(\underline{m}+\mu \kappa) \underline{\underline{m}}(\mu(1+\kappa)-s)^{\mu+p}} \\
& { }_{2} F_{1}\left(\underline{m}, \mu+p ; \mu ; \frac{\mu^{2} \kappa(\mu \kappa+\underline{m})^{-1}}{[\mu-s /(1+\kappa)]}\right),
\end{aligned}
$$

where it is assumed that the $\kappa-\mu$ shadowed fading model is power normalized, and the parameter $\underline{m}$ inherent to the $\kappa-\mu$ shadowed distribution is underlined in order not to be confused with that of the IG distribution. This allows to derive the PDF and CDF of all the composite models arising from the $\kappa-\mu$ shadowed fading model in a straightforward manner.

In other cases, the GMGF of the fading model is readily available in the literature for special values of $m$. This is the case for instance of more sophisticated fading models such as Beckmann or TWDP [28, 29] under the assumption of $m$ being a positive integer. Strikingly, even though neither the Beckmann nor the TWDP distributions admit closed-form expressions for their PDF or CDF, their respective composite models do. Hence, somehow counterintuitively, the IG distribution not only renders more general models, but at the same time their mathematical complexity is relaxed. Moreover, if we assume $m \in \mathbb{N}^{+}$(or, equivalently, $n \in \mathbb{N}^{+}$), the CDF expression in (15) simplifies to a finite sum of evaluations of the GMGF of $X$, as stated next:

Corollary 1: Let $W$ be a RV characterizing the instantaneous received signal power in (9), and assume $m$ is a positive integer, i.e $m \in \mathbb{N}^{+}$. Then, the CDF of $W$ is expressed as

$$
F_{W}(u)=\sum_{n=0}^{m-1} \frac{(m-1)^{n} \bar{W}^{n}}{u^{n} \Gamma(n+1)} \phi_{X}^{(n)}\left(\frac{(1-m) \bar{W}}{u}\right) .
$$

Proof: Substituting in (17) the CDF of the gamma distribution for integer shape parameter, $m$, which is given by

$$
F_{1 / \xi}(t)=1-\sum_{n=0}^{m-1} \frac{(m-1)^{n} t^{n}}{\Gamma(n+1)} e^{(1-m) t},
$$

and following the same steps as in Lemma 2, the proof is completed.

Note that restricting the parameter $m$ to take positive integer values may have limited impact in practice from a goodnessof-fit perspective, unless the shadowing variance is very large.

Last, but not least, the use of the IG distribution to model shadow fading has an additional advantage. It is well-known that the PDF of numerous fading models can be expressed in terms of a mixture of gamma distributions as [30, eq. (1)]:

$$
f_{X}(u)=\sum_{i=1}^{N} w_{i} f_{i}^{\mathrm{G}}\left(k_{i}, \Omega_{i} ; u\right)
$$


where $f_{i}^{\mathrm{G}}(\cdot)$ is the gamma PDF in (2), $w_{i}$ for $i=1, \ldots, N$ are constants and $k_{i}$ and $\Omega_{i}$ are the parameters of the $i$-th gamma distribution, which are obtained by using PDF or MGF matching. In some cases, this mixture form naturally arises by inspection [31, 32]. In all cases on which the PDF of the fading model can be expressed as a mixture of gamma distributions, the PDF of the IG-based composite fading model is directly obtained as a mixture of $\mathcal{F}$ distributions as stated in the following corollary:

Corollary 2: Let $X$ be a RV characterizing the fast fading with PDF as in (22). Then, the PDF of the instantaneous received signal power $W=\xi X$ is given by

$$
f_{W}(u)=\sum_{i=1}^{N} w_{i} f_{\mathcal{F}}\left(m, k_{i}, \Omega_{i} ; u\right),
$$

where $f_{\mathcal{F}}(\cdot)$ is the PDF of the $\mathcal{F}$ distribution [33, eq. (6)]:

$$
f_{\mathcal{F}}(m, k, \Omega ; t)=\frac{(m-1)^{m} k^{k}}{B(m, k)} \frac{t^{k-1} \Omega^{m}}{((m-1) \Omega+k t)^{m+k}}
$$

with $B(\cdot, \cdot)$ the beta function [23, eq. (6.2.2)].

Proof: From (22), the PDF of $W$ is calculated as

$$
f_{W}(u)=\sum_{i=1}^{N} w_{i} \int_{0}^{\infty} t f_{i}^{\mathrm{G}}\left(k_{i}, \Omega_{i} ; u t\right) f_{1 / \xi}(t) d t,
$$

with $f_{i}^{\mathrm{G}}(\cdot)$ as in (2) and $f_{1 / \xi}(t)=f_{1 / \xi}^{\mathrm{G}}(m, m /(m-1) ; t)$. Using [34, eq. (3.381 4)] and performing some algebraic manipulations, the proof is completed.

The above corollary provides a remarkable result, since if the underlying fading model $X$ can be expressed as in (22), then the statistical characterization of the composite model is straightforward, as we can leverage all the existing results given for the simple $\mathcal{F}$ distribution.

Finally, note that although we give expressions for the main statistics of the received signal power $W$, the PDF and CDF of the received signal amplitude $R$ can be straightforwardly derived from (11) and (15) through a change of variables, obtaining $f_{R}(r)=2 r f_{W}\left(r^{2}\right)$ and $F_{R}(r)=F_{W}\left(r^{2}\right)$.

\section{APPLiCATION To TWDP FADING MODEL}

We now aim to provide a simple example to illustrate the usefulness of our approach. The case of the TWDP fading model is considered, which assumes the presence of two dominant specular components and accurately fits field measurements in a variety of propagation scenarios [4].

\section{A. TWDP fading distribution}

According to the TWDP fading model, the received signal power $W_{T}$ is described as:

$$
W_{T}=\left|V_{1} e^{j \varphi_{1}}+V_{2} e^{j \varphi_{2}}+Z\right|^{2},
$$

where $V_{1}$ and $V_{2}$ are constants representing the amplitude of each specular component, $\varphi_{i}$ and $\varphi_{2}$ are RVs following a uniform distribution, i.e. $\varphi_{1,2} \sim \mathcal{U}[0,2 \pi)$ and $Z$ is a complex Gaussian RV such that $Z \sim \mathcal{C N}\left(0,2 \sigma^{2}\right)$. It is assumed that

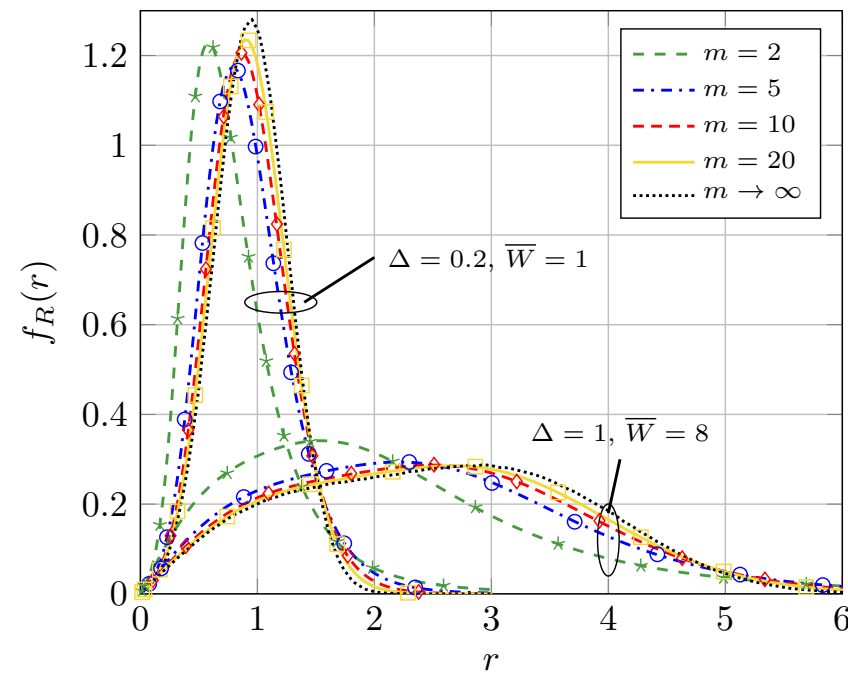

Fig. 4. PDF of the received signal amplitude under inverse gamma / TWDP fading for $K=4$ and different values of $m, \Delta$ and $\bar{W}$. Solid lines correspond to theoretical calculations, markers correspond to MC simulations.

all the involved RVs are statistically independent. The model is completely described by the parameters

$$
K=\frac{V_{1}^{2}+V_{2}^{2}}{2 \sigma^{2}}, \quad \Delta=\frac{2 V_{1} V_{2}}{V_{1}^{2}+V_{2}^{2}},
$$

and its PDF is only known in integral form [4, eq. (7)].

\section{B. Inverse gamma/TWDP composite fading model}

With the TWDP as baseline model, the composite fading model is built as $W=\bar{W} \xi W_{T}$, where $\xi$ follows an inverse gamma distribution as in (9) and $\mathbb{E}\left[W_{T}\right]=1$. In this case, a closed-form expression for the GMGF (10) is known when $n \in \mathbb{N}^{+}$. Therefore, we can directly apply (11) and (20) to characterize $W$, resulting:

$$
\begin{aligned}
f_{W}(u) & =\frac{\bar{W}^{m}(m-1)^{m}}{u^{m+1} \Gamma(m)} \phi_{W_{T}}^{(m)}\left(K, \Delta ; \frac{(1-m) \bar{W}}{u}\right), \\
F_{W}(u) & =\sum_{n=0}^{m-1} \frac{(m-1)^{n} \bar{W}^{n}}{u^{n} \Gamma(n+1)} \phi_{W_{T}}^{(n)}\left(K, \Delta ; \frac{(1-m)}{u \bar{W}^{-1}}\right),
\end{aligned}
$$

where $\phi_{W_{T}}^{(m)}(\cdot)$ is obtained from [29, eq. (4)] with $\bar{\gamma}=1$. For arbitrary $m$, the IG/TWDP composite fading model can be expressed as an infinite mixture of $F$-distributions using [31] and Corollary 2. The expressions for the PDFs and CDFs are not explicitly reproduced here for the sake of compactness.

In order to visualize the impact of $m$ in the distribution of $W$, we show in Figs. 4-5 the PDF of the received signal amplitude calculated from (28) by performing the corresponding change of variables, and contrasted the results with Monte Carlo (MC) simulations. Besides, in order for these PDFs not to be overlapped in the figures, we set different values of $\bar{W}$ for each situation. As expected, with independence of the parameters of the TWDP model, smaller values of $m$ render more spread PDFs, since shadowing can be seen as an increment in the variance of the fast fading model. In turn, 


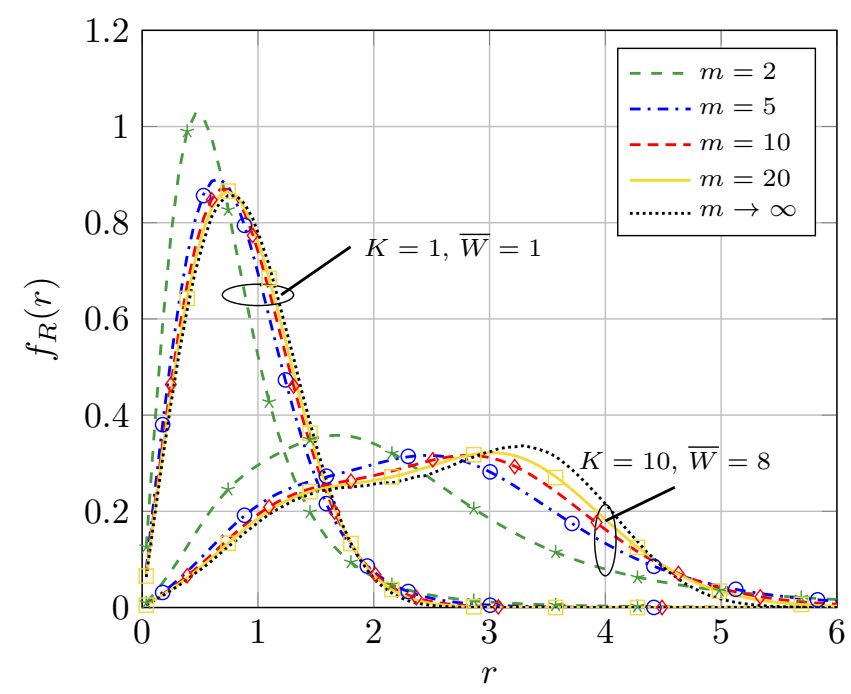

Fig. 5. PDF of the received signal amplitude under inverse gamma / TWDP fading for $\Delta=0.9$ and different values of $m, K$ and $\bar{W}$. Solid lines correspond to theoretical calculations, markers correspond to $\mathrm{MC}$ simulations.

large values of $m$ reduce the shadowing severity and, in the limit $(m \rightarrow \infty)$, the composite model converges to the baseline fading model.

\section{CONCLUSIONS}

We showed that the use of the inverse gamma distribution in the context of composite fading modeling is justified both from empirical evidences and for mathematical tractability. We also provided a general way of deriving the composite fading distribution statistics, which in many cases can be carried out by directly leveraging existing results in the literature.

\section{REFERENCES}

[1] M. K. Simon and M. S. Alouini, Digital communication over fading channels. John Wiley \& Sons, 2005, vol. 95.

[2] G. L. Stuber, "Principles of Mobile Communication", 2nd ed. Kluwer Academic Publishers, 2002.

[3] M. Nakagami, "The $m$-distribution - a general formula of intensity distribution of rapid fading," Stat. Meth. Radio Wave Propag., vol. 47, Dec. 1960.

[4] G. D. Durgin, T. S. Rappaport, and D. A. de Wolf, "New analytical models and probability density functions for fading in wireless communications," IEEE Trans. Commun., vol. 50, no. 6, pp. 1005-1015, Jun. 2002.

[5] M. D. Yacoub, "The $\kappa-\mu$ distribution and the $\eta-\mu$ distribution," IEEE Antennas Propag. Mag., vol. 49, no. 1, pp. 68-81, Feb. 2007.

[6] H. Hashemi, "The indoor radio propagation channel," Proc. IEEE, vol. 81 , no. 7, pp. 943-968, Jul. 1993.

[7] A. Abdi, W. C. Lau, M. . Alouini, and M. Kaveh, "A new simple model for land mobile satellite channels: first- and second-order statistics," IEEE Trans. Wireless Commun., vol. 2, no. 3, pp. 519-528, May 2003.

[8] S. K. Yoo, N. Bhargav, S. L. Cotton, P. C. Sofotasios, M. Matthaiou, M. Valkama, and G. K. Karagiannidis, "The $\kappa-\mu$; / inverse gamma and $\eta-\mu ; /$ inverse gamma composite fading models: Fundamental statistics and empirical validation," IEEE Trans. Commun., pp. 1-1, 2018.

[9] J. F. Paris, "Statistical characterization of $\kappa-\mu$ shadowed fading," IEEE Trans. Veh. Technol., vol. 63, no. 2, pp. 518-526, Feb. 2014.

[10] P. Ramirez-Espinosa, F. J. Lopez-Martinez, J. F. Paris, M. D. Yacoub, and E. Martos-Naya, "An extension of the $\kappa-\mu$ shadowed fading model: Statistical characterization and applications," IEEE Trans. Veh. Technol., vol. 67, no. 5, pp. 3826-3837, May 2018.

[11] C. Loo, "A statistical model for a land mobile satellite link," IEEE Trans. Veh. Technol., vol. 34, no. 3, pp. 122-127, Aug. 1985.
[12] A. Abdi and M. Kaveh, "On the utility of gamma PDF in modeling shadow fading (slow fading)," in IEEE 49th Veh. Technol. Conf., vol. 3, May 1999 , pp. 2308-2312.

[13] — "A comparative study of two shadow fading models in ultrawideband and other wireless systems," IEEE Trans. Wireless Commun., vol. 10, no. 5, pp. 1428-1434, May 2011.

[14] — _ "K distribution: an appropriate substitute for Rayleigh-lognormal distribution in fading-shadowing wireless channels," Electron. Lett., vol. 34, no. 9, pp. 851-852, Apr. 1998.

[15] P. S. Bithas, "Weibull-gamma composite distribution: alternative multipath/shadowing fading model," Electron. Lett., vol. 45, no. 14, pp. 749 -751 , Jul. 2009.

[16] H. Al-Hmood and H. S. Al-Raweshidy, "Unified modeling of composite $\kappa-\mu /$ gamma, $\eta-\mu /$ gamma, and $\alpha-\mu /$ gamma fading channels using a mixture gamma distribution with applications to energy detection," IEEE Antennas Wireless Propag. Lett., vol. 16, pp. 104-108, 2017.

[17] N. Simmons, C. R. N. da Silva, S. L. Cotton, P. C. Sofotasios, and M. D. Yacoub, "Double Shadowing the Rician Fading Model," IEEE Wireless Commun. Lett., vol. 8, no. 2, pp. 344-347, Apr. 2019.

[18] Karmeshu and R. Agrawal, "On efficacy of rayleigh-inverse gaussian distribution over k-distribution for wireless fading channels," Wirel. Commun. Mob. Com., vol. 7, pp. 1-7, 2007.

[19] T. Eltoft, "The rician inverse Gaussian distribution: a new model for non-Rayleigh signal amplitude statistics," IEEE Trans. Image Process., vol. 14 , no. 11 , pp. 1722-1735, Nov. 2005.

[20] P. C. Sofotasios, T. Tsiftsis, K. Ho Van, S. Freear, L. Wilhelmsson, and M. Valkama, "The $\kappa-\mu /$ ig composite statistical distribution in RF and FSO wireless channels," 38th IEEE Veh. Technol. Conf., pp. 1-5, Sep. 2013.

[21] S. K. Yoo, S. L. Cotton, P. C. Sofotasios, M. Matthaiou, M. Valkama, and G. K. Karagiannidis, "The Fisher-Snedecor $\mathcal{F}$ distribution: A simple and accurate composite fading model," IEEE Commun. Lett., vol. 21, no. 7, pp. 1661-1664, Jul. 2017.

[22] P. S. Bithas, A. G. Kanatas, and D. W. Matolak, "Exploiting shadowing stationarity for antenna selection in V2V communications," IEEE Trans. Veh. Technol., vol. 68, no. 2, pp. 1607-1615, Feb. 2019.

[23] M. Abramowitz, I. A. Stegun et al., Handbook of Mathematical Functions with Formulas, Graphs, and Mathematical Tables. Dover, New York, 1972, vol. 9.

[24] M. A. Stephens, "Edf statistics for goodness of fit and some comparisons," J. Am. Stat. Assoc., vol. 69, no. 347, pp. 730-737, 1974.

[25] M. Barbiroli, F. Fuschini, G. Tartarini, and G. E. Corazza, "Smart metering wireless networks at $169 \mathrm{MHz}$," IEEE Access, vol. 5, pp. 83578368, 2017.

[26] B. Ai, K. Guan, R. He, J. Li, G. Li, D. He, Z. Zhong, and K. M. S. Huq, "On indoor millimeter wave massive MIMO channels: Measurement and simulation," IEEE J. Sel. Areas Commun., vol. 35, no. 7, pp. 1678-1690, Jul. 2017.

[27] N. Bhargav, C. R. N. da Silva, S. L. Cotton, P. C. Sofotasios, and M. D. Yacoub, "On shadowing the $\kappa-\mu$ fading model," arXiv preprint arXiv:1808.05013, 2018.

[28] J. P. Peña-Martín, J. M. Romero-Jerez, and F. J. Lopez-Martinez, "Generalized MGF of Beckmann fading with applications to wireless communications performance analysis," IEEE Trans. Commun., vol. 65, no. 9 , pp. 3933-3943, Sep. 2017.

[29] — "Generalized MGF of the two-wave with diffuse power fading model with applications," EEE Trans. Veh. Technol., vol. 67, no. 6, pp. 5525-5529, Jun. 2018.

[30] S. Atapattu, C. Tellambura, and H. Jiang, "A mixture gamma distribution to model the SNR of wireless channels," IEEE Trans. Wireless Commun., vol. 10, no. 12, pp. 4193-4203, Dec. 2011.

[31] N. Y. Ermolova, "Capacity analysis of two-wave with diffuse power fading channels using a mixture of gamma distributions," IEEE Commun. Lett., vol. 20, no. 11, pp. 2245-2248, Nov 2016.

[32] F. J. Lopez-Martinez, J. F. Paris, and J. M. Romero-Jerez, "The $\kappa$ - $\mu$ shadowed fading model with integer fading parameters," IEEE Trans. Veh. Technol., vol. 66, no. 9, pp. 7653-7662, Sep. 2017.

[33] S. K. Yoo, P. C. Sofotasios, S. L. Cotton, S. Muhaidat, F. J. LopezMartinez, J. M. Romero-Jerez, and G. K. Karagiannidis, "A comprehensive analysis of the achievable channel capacity in $\mathcal{F}$ composite fading channels," IEEE Access, vol. 7, pp. 34 078-34 094, 2019.

[34] I. S. Gradshteyn and I. M. Ryzhik, Table of Integrals, Series, and Products. Academic Press, 2007. 